\title{
Cydnidae (Hemiptera: Heteroptera) del Centro de Investigaciones Costeras La Mancha, Actopan, Veracruz, México
}

\section{Cydnidae (Hemiptera: Heteroptera) from the Centro de Investigaciones Costeras La Mancha, Actopan, Veracruz, México}

\author{
Cristina Mayorga-Martínez ${ }^{1 *}$ y Luis Cervantes-Peredo ${ }^{2}$ \\ ${ }_{1}^{1}$ Departamento de Zoología, Instituto de Biología, Universidad Nacional Autónoma de México, Apartado Postal 70-153, México, D.F. \\ ${ }^{2}$ Instituto de Ecología, A.C., Km 2.5 Antigua Carretera a Coatepec 351, Xalapa, Veracruz \\ * Correspondencia: mayorgac@ibiologia.unam.mx
}

\begin{abstract}
Resumen. Se citan 10 especies de Cydnidae (Hemiptera:Heteroptera) presentes en el área del Centro de Investigaciones Costeras La Mancha (CICOLMA) perteneciente al Instituto de Ecología, A.C. en la costa del estado de Veracruz. Se registran 5 géneros: Amnestus, Cyrtomenus, Dallasiellus, Melanaethus y Pangaeus y se describen 2 especies nuevas, Dallasiellus rugosus sp. nov. y Melanaethus dunensis sp. nov. La mayoría de las especies fueron recolectadas bajo la copa de árboles de Ficus spp., alimentándose de los frutos y semillas. Se redescriben brevemente las especies ya descritas, y se reúnen notas acerca de su biología y distribución en el área de estudio y en el país; se incluye una ilustración de cada especie y una clave para su identificación.
\end{abstract}

Palabras clave: Cydnidae, Ficus, Veracruz, México.

\begin{abstract}
Ten species of Cydnidae (Hemiptera: Heteroptera) from the area of the Centro de Investigaciones Costeras La Mancha (CICOLMA), which belongs to Instituto de Ecología, A.C. on the coast of Veracruz are cited. Five genera are registered: Amnestus, Cyrtomenus, Dallasiellus, Melanaethus y Pangaeus and 2 new species are described, Dallasiellus rugosus sp. nov., and Melanaethus dunensis sp. nov. Most of the species were collected under the crown of Ficus spp. trees, feeding on fruits and seeds. A brief redescription of each species, and notes of their biology and distribution in the study area and in the country are included; each species is illustrated and an identification key is also provided.
\end{abstract}

Key words: Cydnidae, Ficus, Veracruz, Mexico.

\section{Introducción}

Las chinches de la familia Cydnidae comúnmente se conocen como chinches excavadoras por su hábito de enterrarse, para lo cual utilizan la cabeza y patas especialmente modificadas. La mayoría de las especies son negras o de color castaño, con la superficie del cuerpo brillante; de forma ovoide a elíptica, y miden entre 1.5 y 17 $\mathrm{mm}$. Varios cídnidos tienen mecanismos de estridulación, donde la vena postcubital y los segmentos del abdomen desempeñan un papel importante. Más de 110 géneros y 600 especies distribuidas en 8 subfamilias se conocen en el mundo; para México se han citado 12 géneros y 39 especies (Schuh y Slater, 1995; Mayorga, 2002).

Poco se conoce sobre la biología de estas especies;

Recibido: 17 marzo 2006; aceptado: 23 junio 2006 algunas se alimentan de las raíces de sus plantas hospederas, viviendo hasta $145 \mathrm{~cm}$ por debajo de la superficie del suelo; otras lo hacen de semillas y jugos de los frutos de sus hospederas que se encuentran sobre el suelo. Muchas especies son atraídas a la luz, siendo su periodo de vuelo muy corto, y en algunas especies parece estar restringido a las primeras horas después de la puesta del sol (Mayorga y Cervantes, 2001; Oliveira et al., 2000; Schuh y Slater, 1995).

En el presente trabajo se describen las especies de Cydnidae presentes en el área del Centro de Investigaciones Costeras La Mancha (CICOLMA) en el estado de Veracruz. Las redescripciones de las especies ya conocidas están basadas en el trabajo de Froeschner (1960). Se describen 2 especies nuevas, una del género Dallasiellus y otra de Melanaethus. Se incluye una clave para su identificación, notas acerca de su biología, distribución y se ilustra cada 
una de ellas.

\section{Material y métodos}

El sitio de estudio (Centro de Investigaciones Costeras La Mancha (CICOLMA)) está situado en la costa del estado de Veracruz, en el municipio de Actopan, $30 \mathrm{~km}$ al NE de Ciudad José Cardel, entre los 96²2'40’' O y 19³6'00’' N. La temperatura media anual es de $25^{\circ} \mathrm{C}$ y la precipitación varia entre 1200 y $1500 \mathrm{~mm}$. La época más seca ocurre entre noviembre y mayo; la temporada de lluvias comienza en junio, con el pico máximo de precipitación en julio (Moreno et al., 1994). En el área se encuentran diversos tipos de vegetación: dunas costeras, manglar, selva baja caducifolia, selva mediana subcaducifolia y selva baja perenifolia inundable (Castillo y Medina, 2002).

Por medio de muestreos mensuales durante $2001 \mathrm{y}$ 2002 y muestreos aislados desde 1999 a la fecha se han recolectado un total de 547 cídnidos. Durante el día, se recolectó por debajo de las plantas hospederas, cavando con una pala pequeña donde se encontraban semillas o frutos. Las chinches se recolectaron manualmente o con la ayuda de un aspirador.

Durante la noche se hicieron recolectas con trampa de luz, utilizando para ello una pantalla de 2 × 2 m iluminada con un foco de luz blanca.

Los organismos recolectados se depositaron en frascos con alcohol al 70\%; posteriormente fueron montados en alfileres entomológicos y por último separados en morfoespecies. Las escalas y medidas se expresan en milímetros. Los ejemplares están depositados en la Colección Nacional de Insectos del Instituto de Biología, UNAM (CNIN), México, D:F., el Instituto de Ecología, A.C. (IEXA) Xalapa, Veracruz, el National Museum of Natural History, Smithsonian, Washington, D.C. (NMNH) y The Natural History Museum (BMNH), Londres.

\section{Descripción.}

\section{Amnestus ficus Mayorga et Cervantes (Fig. 1)}

De 2.8 a 3 mm de largo. Cabeza, pronoto, escutelo y carina prosternal castaño obscuro; carina marginal del pronoto, clavus, corium y vientre abdominal castaño claro; antenas, rostro y patas amarillo pálido. Jugum con 5 espinas, parte posterior del pronoto ligeramente elevada; margen ventral de la carina prosternal recta. Parte posterior de la cabeza con numerosas puntuaciones; tylus más largos que los juga con 4 espinas robustas en el ápice; rostro alcanzando el metasterno. Borde anterior y posterior del pronoto cóncavos, bordes anterolaterales carinados. Tibias

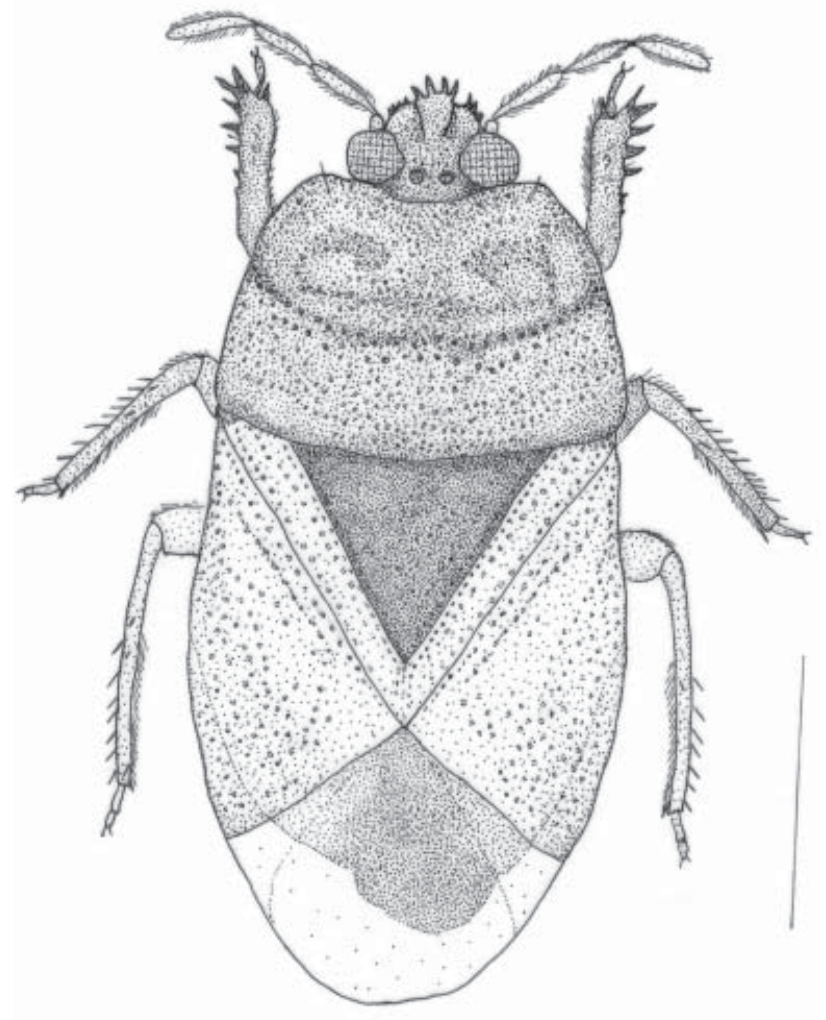

Figura l. Macho de Amnestus ficus Mayorga et Cervantes. Escala $=1 \mathrm{~mm}$.

anteriores ligeramente aplanadas con 7 espinas en su margen externo y 3 en su margen interno; profémur con una espina bífida en la mitad de la superficie ventral; margen interno del metafémur aserrado y con una espina pequeña y bífida cerca de su ápice. Escutelo con puntuaciones distribuidas al azar. Puntuaciones de los hemiélitros distribuidas uniformemente; clavus con 3 hileras de puntuaciones; membrana translucida, extendiéndose más allá del ápice del abdomen.

Biología. Ninfas y adultos de esta especie se encontraron asociadas con Ficus cotinifolia H.B.K y F. trigonata L.f.; y en F. insipida Willd. sólo se encontraron adultos. Su ciclo de vida es muy corto, de aproximadamente 25 días, y se le puede encontrar asociada con otros cídnidos como Pangaeus aethiops (Fab.) y Cyrtomenus crassus Walker. La descripción de los estados inmaduros y más datos de su biología pueden encontrarse en Mayorga y Cervantes (2001).

Distribución. En la zona de estudio se encontró en diversos tipos de vegetación, incluyendo selva baja caducifolia, selva mediana subcaducifolia y selva baja perenifolia 


\section{Clave para las especies de Cydnidae presentes en la Estación CICOLMA}

1. Clavus por debajo del escutelo y formando una comisura casi tan larga como el escutelo; esternitos III y IV sin tricobotrios, esternitos V a VII cada uno con un tricobotrio situado posterior al espiráculo; tercio anterior del peritremo osteolar modificado apicalmente en un anillo, lóbulo o banda bien diferenciado, más ancho que la base del peritremo y más o menos brillante; individuos de menos de $3.5 \mathrm{~mm}$ de longitud

1'. Clavus no por debajo del escutelo y no formando una comisura claval; esternitos III a VII cada uno con 2 tricobotrios localizados variablemente en cada esternito; tercio anterior del peritremo osteolar sin una estructura agrandada o diferenciada apicalmente, algunas veces con una proyección subapical posterior en forma de gancho o ala; individuos mayores a $5 \mathrm{~mm}$ de longitud

2. Protibia distintivamente angulada o con 5 espinas en el margen externo y ninguna en el margen interno; juga con 4 espinas marginales .....................................................................................................mnestus pusio 2'. Protibia no distintivamente angulada, con 7 espinas en su margen externo y 3 espinas en el margen interno; juga con 5 espinas marginales

Amnestus ficus

3. Proceso terminal del peritremo plano y simple, expandido posteriormente como un lóbulo o aurícula más o menos brillante, abertura posterior del osteolo no conspicua ventralmente ............. Melanaethus dunensis sp. nov. 3'. Proceso terminal del peritremo en forma de gancho; con el lóbulo o aurícula opaco, abertura posterior del osteolo conspicua

4. Pronoto anteriormente con una línea profunda, claramente delimitada y corriendo transversalmente a través de los ángulos anteriores (esta línea generalmente sin puntuaciones) 4'. Pronoto anteriormente sin esta línea impresa, aunque frecuentemente con una hilera de puntuaciones en la misma área (raramente con una línea parcial y vaga lateralmente)

5. Evaporitrum de la mesopleura extendiéndose ininterrumpidamente a lo largo del margen posterior del esclerito hasta el ángulo posterolateral .........................................................................................Pangaeus aethiops 5'. Evaporitrum de la mesopleura limitado, separado del ángulo posterolateral y del margen posterior del esclerito por un área brillante

6. Jugum con una puntuación setígera inmediatamente anterior al ojo; dorso de la cabeza fuertemente convexa, usualmente con rugosidades transversas bien marcadas ..............................................Pangaeus rugiceps 6'. Jugum con 3 o 5 puntuaciones setígeras submarginales; superficie de la cabeza no fuertemente convexa, ni marcadamente rugosa Pangaeus bilineatus

7. Metatibia conspicuamente comprimida, caras anterior y posterior glabras no espinadas; espinas del margen posteroventral conspicuamente más largas, delgadas y angostándose más que las del margen dorsal

7'. Metatibia no comprimida o sólo ligeramente, espinas ventrales y dorsales igualmente desarrolladas

Cyrtomenus crassus (Dallasiellus) 8

8. Superficie de la cabeza con abundantes puntuaciones y con 4 a 6 espinas robustas en cada jugum

8'. Superficie de la cabeza sin puntuaciones y 4 a 6 sedas largas en cada jugum Dallasiellus rugosus sp.nov.

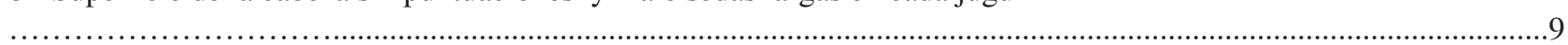

9. Margen de los juga con una carina marginal dorsal que va de los ojos al ápice . Dallasiellus lugubris

9'. Margen de los juga grueso, calloso, sin carina o con una carina parcial submarginal que no llega a los ojos .... Dallasiellus reflexus 
inundable. Se ha registrado en los estados de Chiapas, Guerrero, San Luis Potosí y Veracruz.

\section{Amnestus pusio (Stål) (Fig. 2)}

Pequeña, de 1.2 a $2.1 \mathrm{~mm}$ de largo. Juga con 4 espinas; con puntuaciones presentes en los callos del pronoto. De color castaño amarillento, lóbulo posterior del pronoto y corium mucho más claros; rostro y patas amarillo pálido. Área entre los ojos fuertemente punteada; rostro llegando a las coxas posteriores. Margen anterior del pronoto cóncavo; márgenes laterales rectos o ligeramente sinuados en su mitad basal; sutura transversa prominente y con una hilera de puntuaciones. Mesocorium con algunas pequeñas puntuaciones aisladas; exocorium fuertemente punteado. Carina prosternal tan alta como el artejo rostral II. Profémur con una espina simple o bífida en su ápice, margen posteroventral con un pequeño tubérculo subapical; protibia sin espinas y no angulada.

Biología. Esta especie comúnmentel es atraída hacia la luz. En varias ocasiones se recolectó en áreas cubiertas de pastos, por lo que probablemente está asociada a éstos.

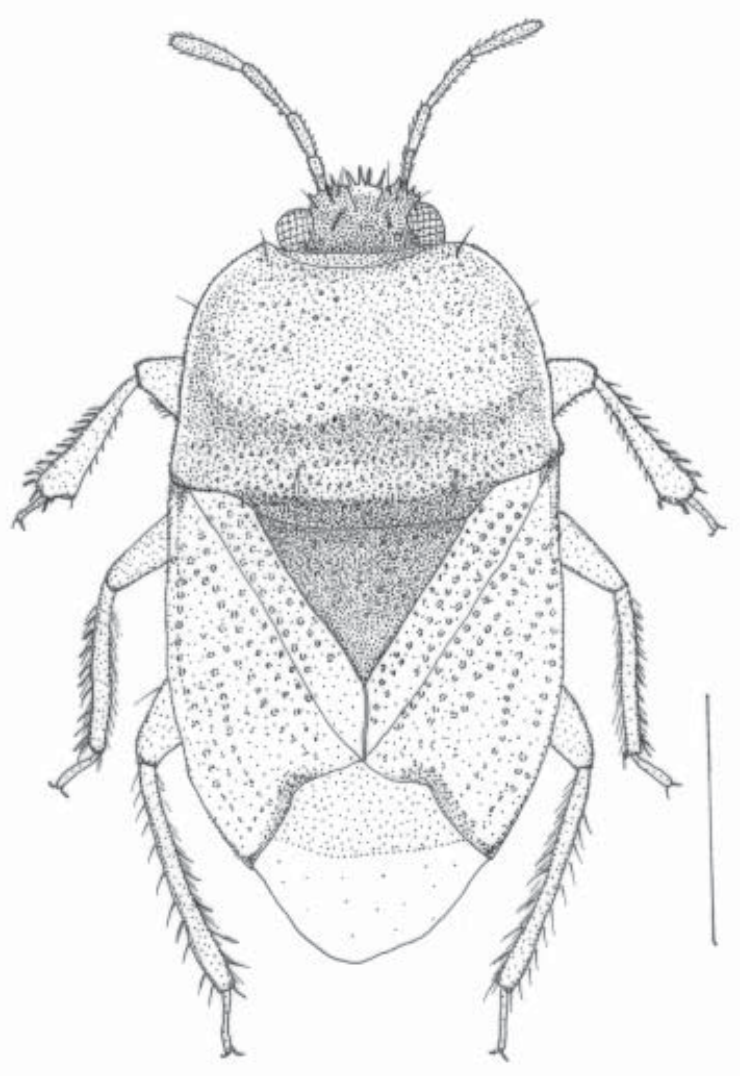

Figura 2. Macho de Amnestus pusio (Stål). Escala $=1 \mathrm{~mm}$.
En una ocasión se recolectaron algunos ejemplares bajo la copa de un árbol de Ficus cotinifolia que se encontraba en fructificación. Se recolectó en marzo y de agosto a noviembre.

Distribución. En la estación se le ha encontrado en la zona de selva baja perenifolia inundable y en la selva mediana subcaducifolia. Se ha registrado en México para Chiapas, Sinaloa y Veracruz. Otros países: Estados Unidos de América, Honduras, Costa Rica, Panamá, Colombia, Brasil, Ecuador, Bahamas, Cuba, Haití, República Dominicana, Puerto Rico, Islas Vírgenes, Granada.

\section{Cyrtomenus (Cyrtomenus) crassus Walker (Fig. 3)}

Parte anterior a los ojos fuertemente redondeada, permitiendo que los ojos sólo sobresalgan ligeramente. Presencia de una hilera postmedia de puntuaciones setígeras en los esternitos IV a VI. De 8.4 a $9 \mathrm{~mm}$ de largo. Ojos proyectados a los lados de la cabeza no más de un tercio de su ancho; juga más largos que el clipeo y más o menos contiguos por enfrente de él; superficie notablemente cóncava, brillante, con prominentes e

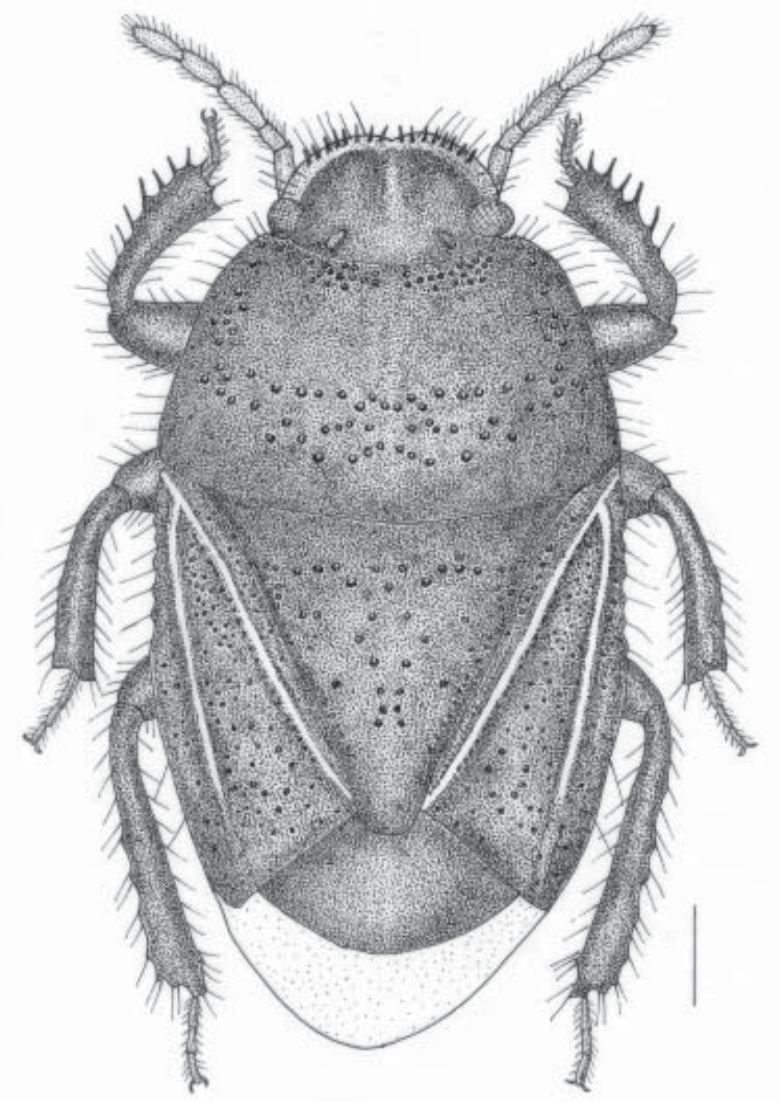

Figura 3. Macho de Cyrtomenus (Cyrtomenus) crassus Walter. Escala $=1 \mathrm{~mm}$. 
irregulares rugosidades, radiando de la base del clipeo; ocelos grandes; juga ventralmente brillante; rostro alcanzando las coxas posteriores. Márgenes laterales del pronoto de rectos a ligeramente cóncavos en los dos tercios basales, con una hilera de 15 a 18 puntuaciones setígeras; lóbulo anterior sin puntuaciones, excepto algunas en los márgenes laterales y en la porción apical; lóbulo posterior con varias puntuaciones situadas anteriormente, siendo más numerosas en la parte media. Escutelo con algunas puntuaciones bien marcadas muy aisladas. Clavus y corium brillantes; clavus con una hilera de puntuaciones; 2 hileras de puntuaciones en el mesocorium paralelas a la sutura claval; exocorium con menos puntuaciones que el mesocorium; costa con 6 a 10 puntuaciones setígeras. Tibias posteriores fuertemente dilatadas hacia el ápice; protibia con 9 o 10 espinas robustas.

Biología. Esta especie se recolectó asociada con Amnestus ficus debajo de Ficus cotinifolia, en mayo, junio, octubre y diciembre.

Distribución. En CICOLMA se recolectó en zonas de selva mediana subcaducifolia.

En México se distribuye en los estados de Baja California, Chihuahua, Coahuila, Guanajuato, Guerrero, Jalisco, Morelos, Nayarit, Oaxaca, Sinaloa y Veracruz. Otros países: Estados Unidos de América, Cuba, Guatemala, Honduras, Costa Rica.

\section{Dallasiellus (Dallasiellus) lugubris (Stål) (Fig. 4)}

El subgénero Dallasiellus se caracteriza por una carina dorsal completa en el margen de la cabeza. Presenta 2 o más puntuaciones setígeras muy contiguas enfrente de los ojos y dos hileras de puntuaciones en el mesocorium, paralelas a la sutura claval.

Mide de 3.9 a $5.5 \mathrm{~mm}$ de largo. Juga ligeramente más largos que el clipeo; superficie pulida, sin puntuaciones y con surcos radiales muy tenues. Búcula tan elevada como el artejo rostral II; rostro llegando a las coxas medias. Pronoto con margen anterior doble emarginado; márgenes laterales enteros, ligeramente curvados en el tercio anterior, con 6 puntuaciones setígeras submarginales; lóbulo anterior con una banda de puntuaciones subapical y con un parche de puntuaciones bien definidas lateralmente; lóbulo posterior con puntuaciones hacia la línea media y lateralmente. Escutelo con numerosas puntuaciones, más finas hacia el ápice. Corium y clavus pulidos; clavus con una hilera completa y una hilera incompleta de puntuaciones; mesocorium con 2 hileras; costa con 1 a 3 puntuaciones setígeras.

Biología. Se encontró en abril, junio, julio, agosto, septiembre, noviembre y diciembre, y se le atrajo a la luz

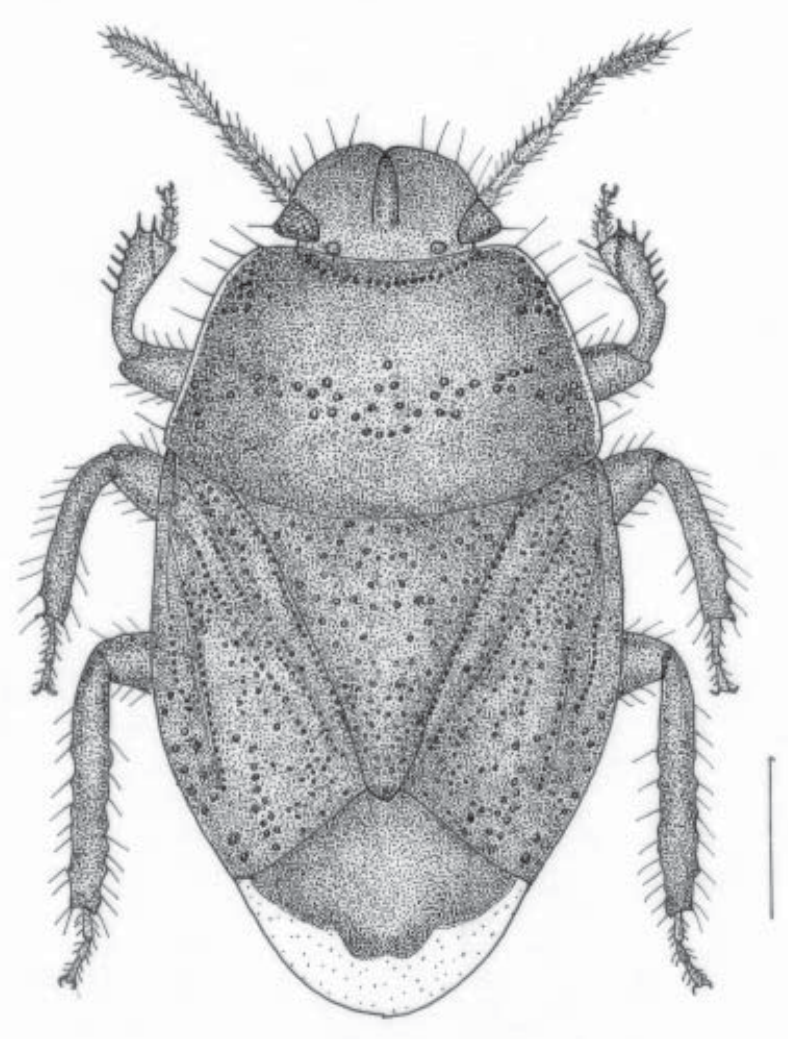

Figura 4. Macho de Dallasiellus (Dallasiellus) lugubris (Stål). Escala $=1 \mathrm{~mm}$.

en el área de los edificios.

Distribución. En México está en Veracruz y Yucatán. Otros países: Guatemala, Nicaragua, Costa Rica, Panamá, Brasil, Bolivia, Argentina, Puerto Rico.

Dallasiellus (Dallasiellus) rugosus sp. nov. Mayorga y Cervantes (Fig. 5)

Las hembras de esta especie son de color pardo oscuro, excepto las antenas, rostro y tarsos de los 3 pares de patas que son de color pardo claro. Cabeza con carina submarginal, y 4 espinas robustas en cada juga. Superficie de todo el cuerpo con numerosas puntuaciones. Cabeza con abundantes puntuaciones en toda su superficie; tylus del mismo tamaño que los juga; búcula elevada; rostro alcanzando el metasterno. Pronoto con el margen anterior cóncavo con una hilera paralela de puntuaciones; bordes anterolaterales carinados con 5 puntuaciones setígeras; sutura media bien marcada con una hilera de puntuaciones; 


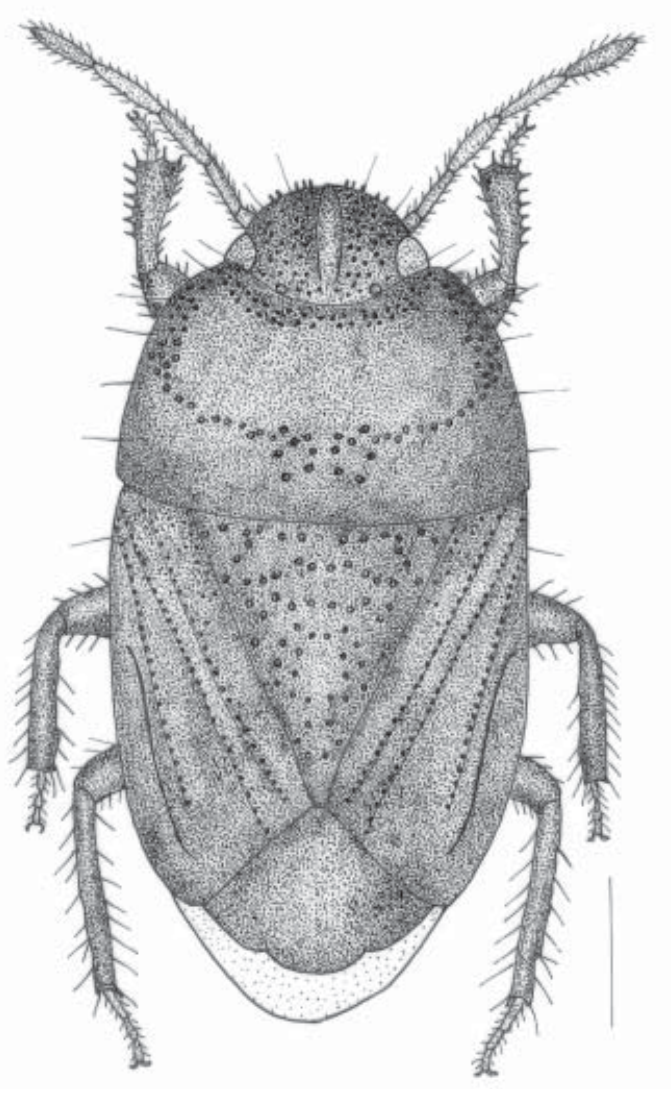

Figura 5. Hembra de Dallasiellus (Dallasiellus) rugosus sp. nov. Mayorga et Cervantes. Escala $=1 \mathrm{~mm}$.

lóbulo anterolateral también con puntuaciones bien marcadas. Escutelo brillante, con puntuaciones dispersas en su superficie. Hemiélitro con corium y clavus con una hilera completa de puntuaciones; área costal con una puntuación setígera evidente; disco del mesocorium con puntuaciones bien marcadas. Patas anteriores con la tibia levemente aplanada, con 7 espinas en su margen externo y dos en el interno.

Medidas de la hembra. Longitud del cuerpo 5.2 \pm 0.2 . Longitud de la cabeza $0.70 \pm 0.1$. Anchura a través de

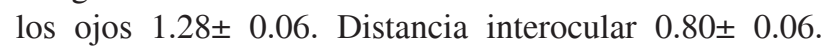
Distancia interocelar $0.43 \pm 0.06$. Artejos antenales: I $0.19 \pm .06$; II $0.31 \pm 0.05$; III $0.34 \pm 0.1$; IV $0.50 \pm 0.06$; V $0.57 \pm 0.06$; artejos rostrales: I $0.52 \pm 0.1$; II $0.62 \pm 0.06$; III $0.52 \pm 0.06$; IV $0.36 \pm 0$. Longitud del pronoto $1.44 \pm 0.1$. Anchura a través del margen anterior 1.48 \pm 0.1 . Anchura a través de los ángulos humerales 2.71 \pm 0.2 . Escutelo: Longitud 1.94 \pm 0.1 . Anchura del escutelo 1.86 \pm 0 . Longitud del profémur $1.02 \pm 0$. Protibia $0.96 \pm 0$. Tarsos anteriores I $0.18 \pm 0$; II $0.12 \pm 0$; III $0.18 \pm 0$. Longitud del metafémur;
$1.52 \pm 0.1$. Metatibia 1.77 \pm 0.6 . Tarsos posteriores I $0.18 \pm 0$; II $0.12 \pm 0$; III $0.18 \pm 0$.

\section{Resumen taxonómico}

Holotipo: Hembra. México Veracruz, Actopan, La Mancha, 28-VIII-1999 L. Cervantes (IEXA).

Paratipos: Misma localidad que el holotipo, 26-XI-1998, L. Cervantes 8 hembra; 28-VIII-1999, L.Cervantes 9 hembra; 13-VI-2000, L.Cervantes 3 hembra (IEXA); 12VIII-2000, Cervantes 5 hembra; 18-VIII-2001, L.Cervantes y A.Sánchez 8 hembra (CNIN); 26-IX-2003, L.Cervantes 19 hembra (CNIN; NMNH; BMNH).

Etimología. Por la apariencia rugosa de la superficie del cuerpo.

Esta especie también se encuentra dentro del subgénero Dallasiellus y se diferencia de Dallasiellus lugubris, por las espinas robustas que se encuentran en los juga y la gran cantidad de puntuaciones en la superficie del cuerpo, características que ninguna otra especie del género presenta.

Biología. Esta especie se recolectó con trampa de luz de junio a noviembre, resaltando que únicamente se recolectaron hembras.

Distribución: México, Veracruz, Actopan, CICOLMA.

\section{Dallasiellus (Ecarinoceps) reflexus Froeschner (Fig. 6)}

El subgénero Ecarinoceps se caracteriza por no tener una carina marginal dorsal en los juga. Esta especie se distingue por la búcula elevada que termina abruptamente en su parte posterior. De 8.24 a $8.85 \mathrm{~mm}$ de largo. Clípeo tan largo como los juga; juga sin puntuaciones con algunas rugosidades tenues y con una puntuación setígera enfrente del ojo; ocelos pequeños. Margen anterior del pronoto moderadamente cóncavo; márgenes laterales enteros; sutura transversa obsoleta o ausente; lóbulo posterior con algunas puntuaciones en su parte media. Escutelo brillante, con puntuaciones excepto en su base y ápice. Corium y clavus pardo claro, exocorium y mesocorium principalmente con puntuaciones muy tenues u obsoletas, mesocorium además con 2 hileras de puntuaciones paralelas a la sutura claval; clavus con una hilera y media de puntuaciones; costa con dos puntuaciones setígeras; patas moderadamente largas.

Biología. Esta especie ha sido recolectada debajo de la copa de Ficus cotinifolia y F. trigonata, durante los meses de marzo, abril, mayo, agosto, septiembre, octubre y noviembre, siendo muy abundantes en agosto.

Distribución. En CICOLMA se recolectó en zonas de selva mediana subcaducifolia y selva baja perenifolia inundable. Se ha registrado para: Colima y Veracruz 


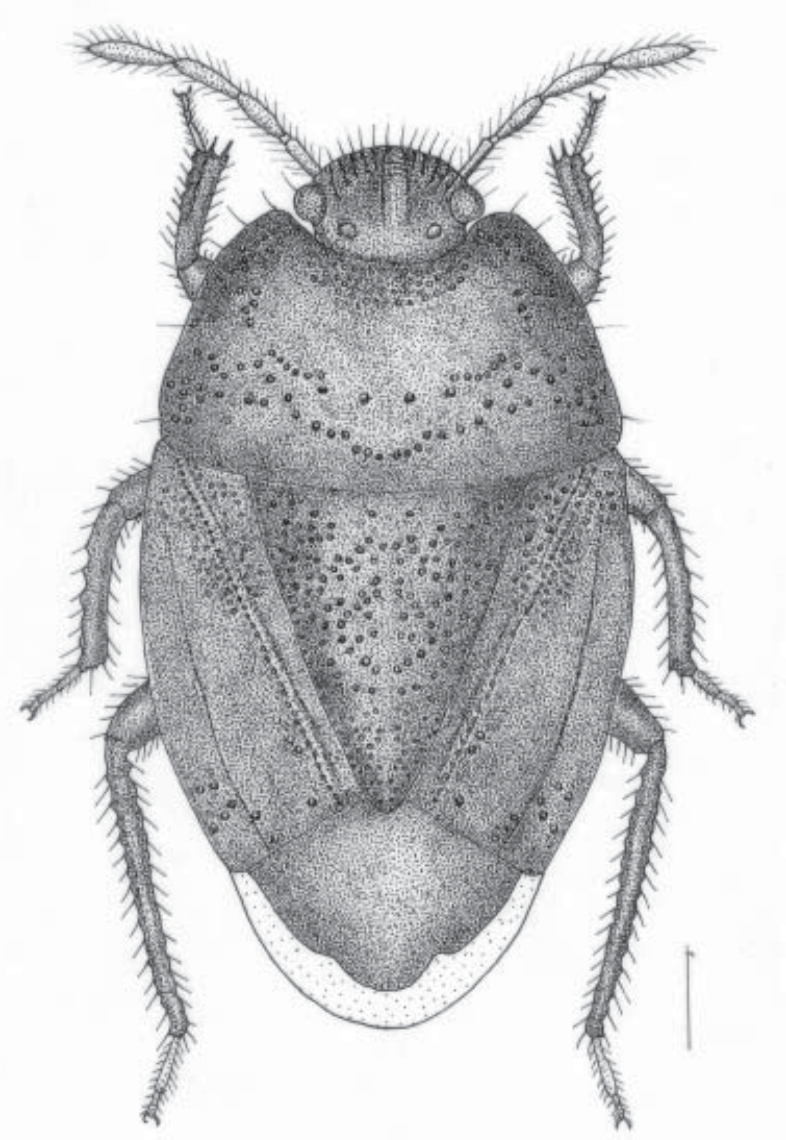

Figura 6. Macho de Dallasiellus (Ecarinoceps) reflexus Froeschner. Escala $=1 \mathrm{~mm}$.

Melanaethus dunensis sp. nov. Mayorga et Cervantes (Fig. 7)

Pequeña, de aproximadamente $3 \mathrm{~mm}$ de largo. Se caracteriza por tener 4 sedas largas en cada margen lateral del pronoto. Se distingue fácilmente de las demás especies del género por la forma del parámero en los machos, el cual tiene 2 prolongaciones bien definidas (Fig. 7a). Oval, más ancho en el tercio posterior. De color negro brillante. Cabeza completamente pegada al margen anterior del pronoto y ligeramente declivente; ojos y ocelos blanquecinos o ligeramente rojizos; algunas puntuaciones ligeramente marcadas en la parte anterior de la cabeza; una puntuación setígera situada sobre el margen lateral y otra situada cerca del margen interno de los ojos; rostrum llegando a las mesocoxas. Pronoto con el margen anterior más o menos cóncavo, con la parte media recta: puntuaciones irregularmente distribuidas, aunque menos abundantes en la parte discal; 4 o puntuaciones setígeras regularmente distribuidas a lo largo de los márgenes laterales. Escutelo

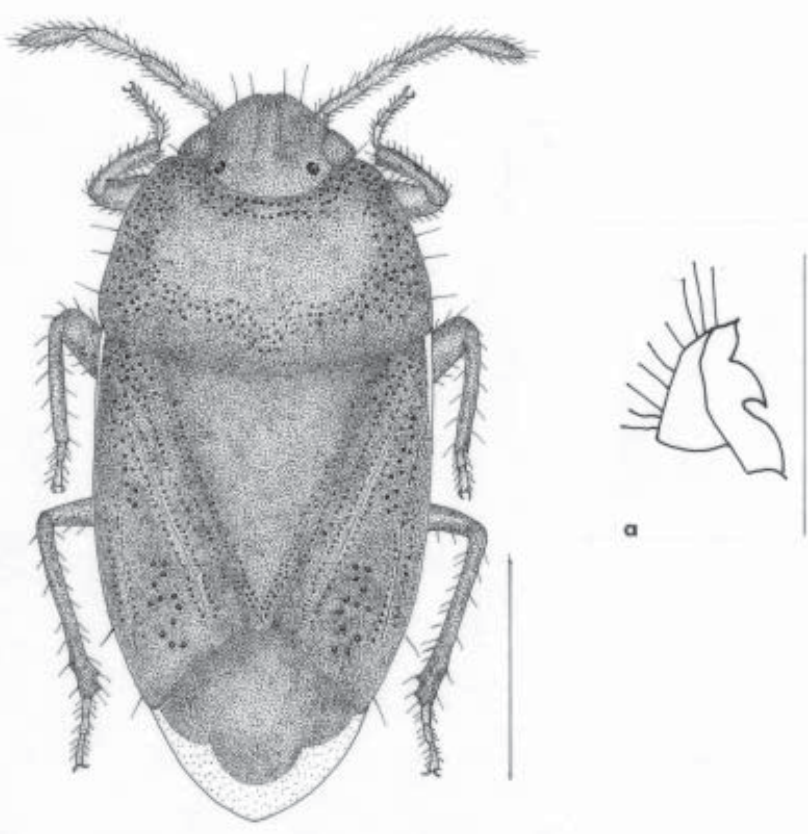

Figura 7. Macho de Melanaethus dunensis sp. nov. Mayorga et Cervantes; a, paramero en vista lateral. Escala $=1 \mathrm{~mm}$.

de ápice redondeado; con numerosas puntuaciones y las situadas en sus 3 márgenes formando una hilera. Clavus y corium con numerosas puntuaciones bien marcadas y arregladas en hileras. Profémur ligeramente ensanchado pero sin espinas. Tibias de todas las patas con numerosas espinas gruesas regularmente distribuidas; protibias con 4 o 5 espinas. Parte anterior de la propleura con algunas puntuaciones ligeramente marcadas. Evaporitrum con superficie rugosa ocupando más o menos la mitad de la mesopleura y más de la mitad de la metapleura; peritremo ligeramente elevado y con el lóbulo terminal redondeado. Esternitos convexos, de color negro brillante. Pigóforo de margen completo y redondeado; parámeros con dos prolongaciones bien definidas.

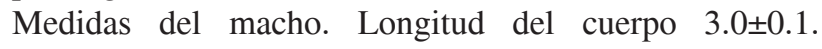
Longitud de la cabeza $0.34 \pm 0$; anchura a través de los ojos $0.72 \pm 0$. Distancia interocular $0.45 \pm 0$. Distancia interocelar $0.31 \pm 0.03$. Artejos antenales: I $0.15 \pm 0$; II $0.11 \pm 0$; III $0.15 \pm 0$ IV $0.22 \pm 0 ; \mathrm{V} 0.26 \pm 0$. Artejos rostrales: I $0.22 \pm 0$; II $0.35 \pm 0.03$; III $0.26 \pm 0.08$; IV $0.20 \pm 0.01$. Longitud del 
pronoto $0.77 \pm 0.03$. Anchura a través del margen anterior $0.70 \pm 0.04$. Anchura a través de los ángulos humerales 1.44 \pm 0 . Escutelo: Longitud $1.17 \pm 0$; anchura $0.87 \pm 0$. Longitud del profémur $0.51 \pm 0.04$. Protibia $0.41 \pm 0$. Tarsos anteriores: I $0.07 \pm 0$; II $0.03 \pm 0$; III $0.11 \pm 0$. Metafémur $0.77 \pm 0.02$. Metatibia $0.89 \pm 0.02$. Tarsos posteriores: I $0.07 \pm 0$; II $0.03 \pm 0$; III $0.11 \pm 0$.

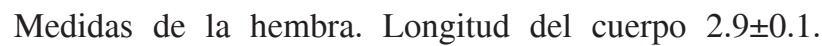
Longitud de la cabeza $0.32 \pm 0.02$. Anchura a través de los ojos $0.72 \pm 0$. Distancia interocular $0.45 \pm 0$. Distancia interocelar $0.33 \pm 0.01$. Artejos antenales: I $0.15 \pm 0$; II $0.11 \pm 0$; III $0.19 \pm 0$; IV $0.22 \pm 0$; V $0.26 \pm 0$. Artejos rostrales: I $0.22 \pm 0$; II $0.38 \pm 0$; III $0.26 \pm 0$; IV $0.19 \pm 0$. Longitud del pronoto $0.79 \pm 0$. Anchura a través del margen anterior $0.76 \pm 0$. Anchura a través de los ángulos humerales $1.44 \pm 0$. Escutelo: Longitud 1.17 \pm 0 ; anchura $0.87 \pm 0$. Longitud del profémur $0.45 \pm 0$. Protibia $0.41 \pm 0$. Tarsos anteriores: I $0.07 \pm 0$; II $0.03 \pm 0$; III $0.11 \pm 0$. Longitud del metafémur $0.76 \pm 0$. Metatibia $0.89 \pm .02$; Tarsos: I $0.07 \pm 0$; II $0.03 \pm 0$; III $0.11 \pm 0$.

\section{Resumen taxonómico}

Holotipo: Macho. México, Veracruz; Actopan, La Mancha, 26-IX-2003, L. Cervantes (IEXA).

Paratipos: Veracruz, Actopan, La Mancha, 26-IX-2003 L.Cervantes 2 machos y 4 hembras. Veracruz, Actopan, La Mancha, 26-IX-2003, T.Mejía, 1 macho (IEXA, CNIN).

Etimología. Derivado del lugar en el que habitan, las dunas costeras.

Biología. Los 8 ejemplares de esta especie fueron recolectados en trampa de luz fluorescente en el área de dunas costeras, en una zona dominada por pastos, durante el mes de septiembre.

Distribución. México, Veracruz, Actopan, CICOLMA.

\section{Pangaeus (Homaloporus) biliniatus (Say) (Fig. 8)}

Esta especie se puede distinguir por la ausencia de tubérculos en la superficie ventral de los fémures posteriores; la ausencia de puntuaciones en la impresión subapical del pronoto, y por la presencia de tres o más puntuaciones setígeras submarginales en cada jugum. De 5.85 a $7.46 \mathrm{~mm}$ de largo. Clípeo tan largo como los juga; juga con 3 a 5 puntuaciones setígeras submarginales, usualmente con rugosidades tenues u obsoletas; ocelos moderadamente grandes; búcula tan elevada como el artejo rostral II; rostro llegando a las coxas medias. Margen anterior del pronoto emarginado; márgenes laterales enteros, casi rectos en su tercio medio con 9 a 12 puntuaciones setígeras; sutura transversa tenue $\mathrm{u}$ obsoleta; lóbulo anterior sin puntuaciones o con algunas

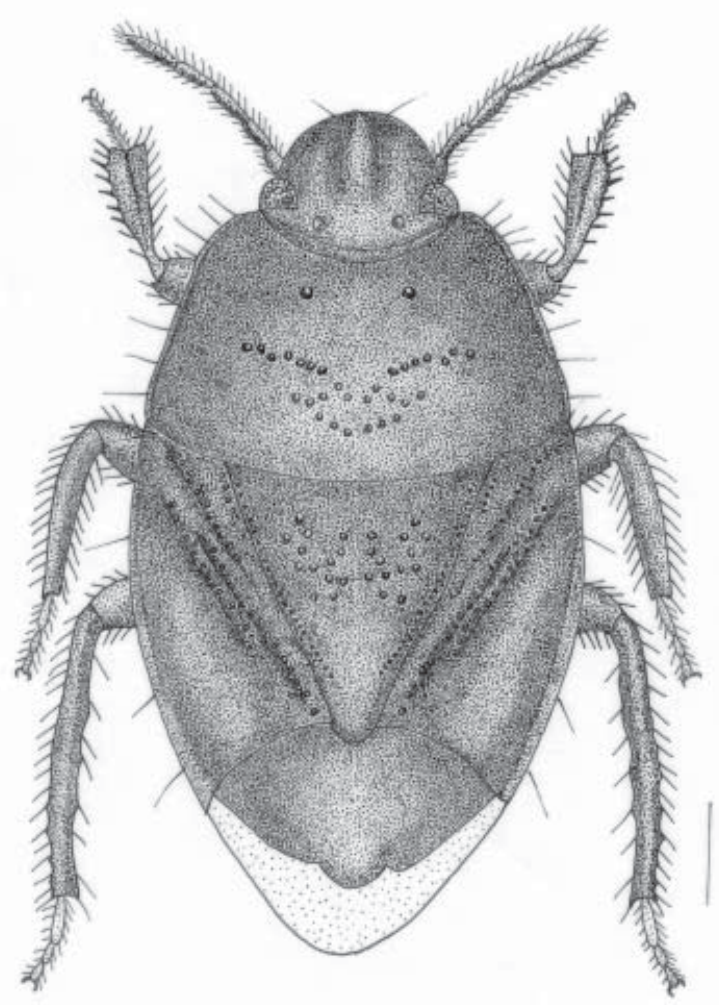

Figura 8. Macho de Pangaeus (Homaloporus) biliniatus (Say). Escala $=1 \mathrm{~mm}$.

lateralmente; lóbulo posterior sin puntuaciones o con algunas en su parte media. Escutelo con puntuaciones, excepto en su base y en el extremo de su ápice. Clavus con 1 o 2 hileras parciales de puntuaciones; mesocorium con una completa y otra completa o incompleta hilera de puntuaciones paralelas a la sutura claval; exocorium con numerosas puntuaciones, más distintivas que aquellas del disco del mesocorium; costa con 2 a 6 puntuaciones setígeras. Patas moderadamente largas; metafémur no tuberculado ventralmente y metatibia no angulada.

Biología. Fue recolectado bajo la copa de Ficus cotinifolia de mayo a noviembre

Distribución. En CICOLMA, se encontró en zonas de selva mediana subcaducifolia y selva baja perenifolia inundable. Se ha registrado para México en Baja California, Coahuila, Durango, Distrito Federal, Guerrero, Hidalgo, Jalisco, Michoacán, Morelos, Puebla, San Luis Potosí, Veracruz. Otros países: Estados Unidos de América, Guatemala. 


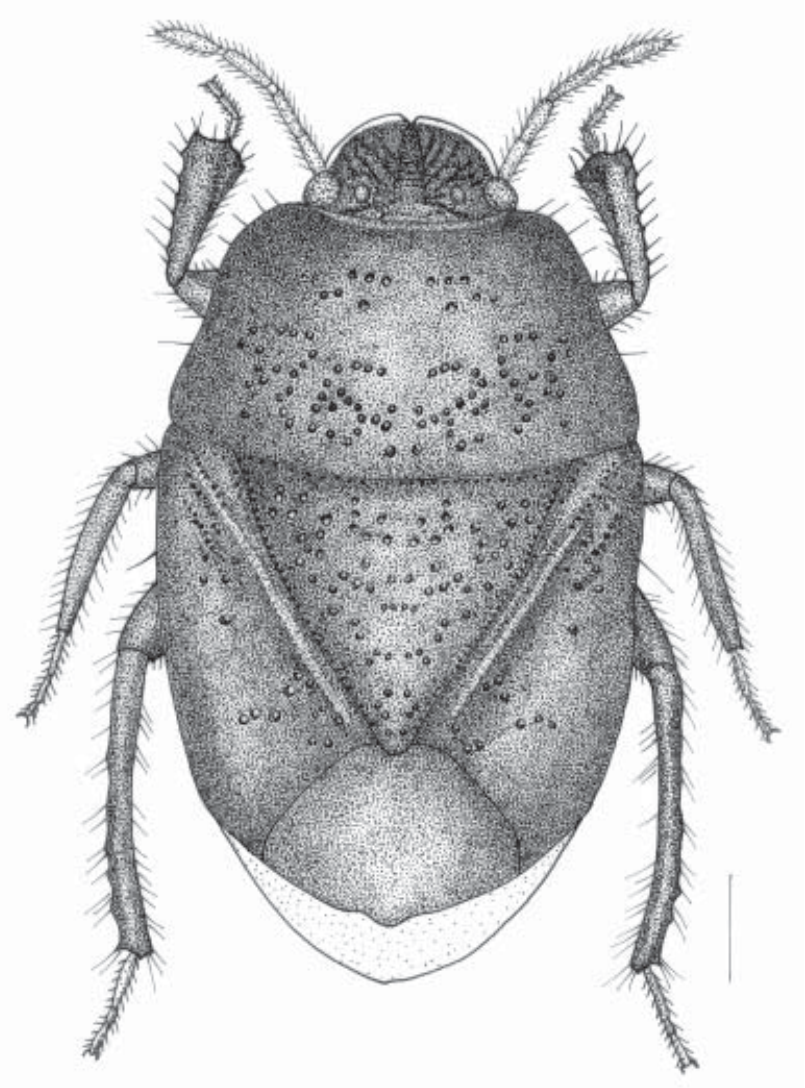

Figura 9. Macho de Pangaeus (Homaloporus) rugiceps Horvath. Escala $=1 \mathrm{~mm}$.

\section{Pangaeus (Homaloporus) rugiceps Horvath (Fig. 9)}

Dentro del subgénero Homaloporus, es la única especie con una sola puntuación setígera por delante de los ojos. Entre 5.6 y 6.7 mm de largo. Jugum más largo que el clipeo, y casi unidos por enfrente de él; juga sin puntuaciones, pero con rugosidades transversas; ocelos moderadamente grandes; búcula tan elavada como la mitad del artejo rostral II; rostro llegando a las coxas medias. Margen anterior del pronoto doblemente emarginado; márgenes laterales de rectos a ligeramente sinuados a la altura de la sutura transversa; sutura transversa tenue, marcada por puntuaciones aisladas; resto de la superficie del pronoto sin puntuaciones, excepto algunas en el margen lateral del lóbulo anterior y en la parte media del lóbulo posterior. Escutelo pulido, con el tercio o cuarto basal sin puntuaciones, disco con algunas puntuaciones aisladas. Clavus y corium brillantes; clavus con una hilera parcial de puntuaciones; mesocorium con 2 hileras completas de puntuaciones: exocorium con algunas tenues puntuaciones en toda su superficie; costa con 2 o

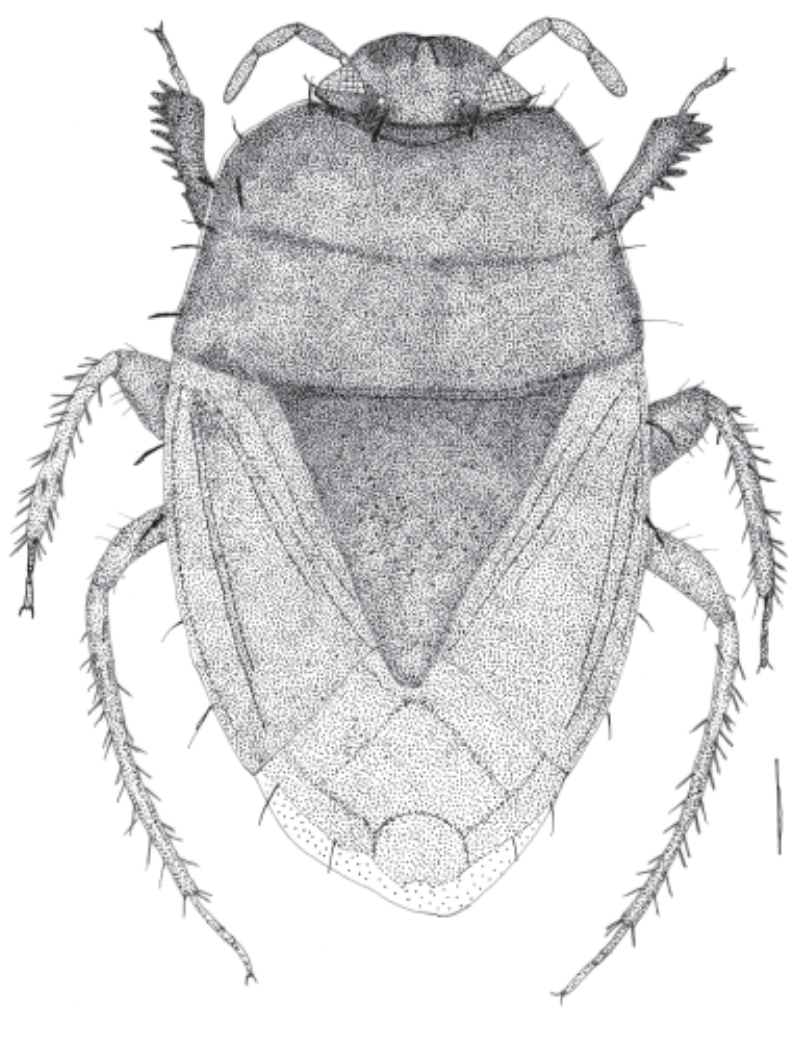

Figura 10. Macho de Pangaeus (Pangaeus) aethiops (Fabricius). Escala $=1 \mathrm{~mm}$.

3 puntuaciones setígeras. Metafémur sin tubérculos; metatibia no angulada en su base.

Biología. Fue recolectada siempre en trampa de luz y estuvo presente de mayo a agosto.

Distribución. En CICOLMA se recolectó en zonas de selva mediana subcaducifolia y selva baja perenifolia inundable.

En México se registra para Chiapas, Colima, Guerrero, Jalisco, Morelos, Nuevo León, Oaxaca, San Luis Potosí, Sinaloa, Sonora y Veracruz. Otros países: Estados Unidos de América, Guatemala.

\section{Pangaeus (Pangaeus) aethiops (Fabricius) (Fig. 10)}

Las especies del género Pangaeus se distinguen por tener una banda transversal paralela al margen anterior del pronoto. Y dentro del género, esta especie se distingue por la emarginación medio apical muy profunda de la cápsula genital. De 7 a 9.45 mm de largo. Juga tan largos o más 
que el clípeo; superficie pulida con algunas puntuaciones pequeñas aisladas; con una puntuación setígera por enfrente de los ojos; ocelos grandes; búcula casi tan elevada como el artejo rostral II; rostro llegando o pasando ligeramente las coxas medias. Margen anterior del pronoto doblemente emarginado; márgenes laterales rectos en sus dos tercios basales con 4 o 5 puntuaciones setígeras; sutura transversa tenue, pero evidente, marcada por una hilera media de puntuaciones; lóbulo anterior con una línea media marcada; lóbulo posterior con muchas puntuaciones en toda su superficie, más abundantes en la parte media. Escutelo pulido, con puntuaciones casi hasta su ápice. Clavus y mesocorium con una hilera completa y algunas veces con una hilera incompleta de puntuaciones; costa con 2 puntuaciones setígeras. Metatibia con el margen posteroventral con 5 tubérculos y con una angulación distintiva en el sexto basal, y 3 o 4 espinas subapicales.

Biología. Ha sido recolectada debajo de la copa de Ficus cotinifolia, F. insipida y $F$. trigonata, fue recolectada desde abril hasta diciembre y es una de las especies más comúnmente atraídas a la luz.

Distribución. En CICOLMA, en selva baja caducifolia, selva mediana subcaducifolia y selva baja perenifolia inundable. Se encuentra registrada para Chiapas, Guerrero, Estado de México, San Luis Potosí, Veracruz y Yucatán. Otros países: Guatemala, Honduras, Nicaragua, Granada, Trinidad, Guiana Francesa, Guiana Británica, Venezuela, Colombia, Ecuador, Brasil, Perú, Paraguay, Bolivia, Argentina, Uruguay.

\section{Comentarios taxonómicos}

La mayoría de las especies pueden encontrarse en las áreas que están cubiertas por selva baja caducifolia, selva mediana subcaducifolia y selva baja perenifolia inundable, en donde están presentes individuos de 4 especies de Ficus, F. cotinifolia, F. insipida, F. obtusifolia y F. trigonata. Las especies más probables de encontrarse aquí son: A. ficus, $D$. reflexus, $P$. aethiops y $P$. bilineatus. Durante la noche es factible encontrar individuos de estas especies y de las restantes 6 en los focos que iluminan los edificios de la Estación CICOLMA, o bien, poniendo una trampa de luz en las áreas de vegetación arriba mencionadas.

Aunque no se conoce todavía la biología de muchas de las especies de cídnidos aquí mencionadas, varias se alimentan con los frutos en descomposición de Ficus spp. que se encuentran en el suelo, succionando los jugos de éstos, o con sus semillas. Otras especies como A. pusio y M. dunensis sp nov., probablemente se alimentan de las raíces de pastos.

Las mayoría de las especies de cídnidos que se encontraron en CICOLMA estuvieron presentes casi todo el año, lo que se debe principalmente a que las especies de Ficus tienen una fructificación asincrónica durante todo el año y las especies de pastos están siempre presentes, lo que proporciona recursos alimenticios abundantes para los cídnidos y, a su vez, significa que los cídnidos en esta zona tienen varias generaciones al año.

\section{Agradecimientos}

Se agradece a la Biól. Iliana Pacheco por la ayuda en la recolecta de algunos ejemplares. Parte del material fue recolectado durante la realización de otro proyecto ("Lygaeidae (Hemiptera-Heteroptera) asociados a Ficus spp. (Moraceae) y su efecto en la depredación de semillas en la Región del Golfo de México") con financiamiento de CONACYT (34238-V), otorgado al segundo autor.

\section{Literatura citada}

Castillo, G. y M. E. Medina. 2002. Árboles y arbustos de la Reserva Natural de La Mancha, Veracruz. Instituto de Ecología, A.C. Xalapa, Veracruz, 144 p.

Froeschner, R. C. 1960. Cydnidae of the Western Hemisphere. Proceedings of the United States National Museum 111: 337-680.

Mayorga, M. M. C. 2002. Revisión genérica de la familia Cydnidae (Hemiptera-Heteroptera) en México, con un listado de las especies conocidas. Anales Instituto de Biología, Universidad Nacional Autónoma de México 73(2): 157-192.

Mayorga, M. M. C. y L. Cervantes. 2001. Life cycle and description of a new species of Amnestus Dallas (Hemiptera-Heteroptera: Cydnidae) associated with the fruit of several species of Ficus (Moraceae) in Mexico. Journal of the New York Entomological Society 109(3-4): 392-402.

Oliveira, L. J., A. B. Malaguido, J, N. Júnior, I. C. Corso, S. De Angelis, L. C. de Faria, C. B. Hoffmann Campo y A. F. Lantmann. 2000. Pecevelo-Castanho da raiz em sistema de producción de soya. EMBRAPA, Londrina. $44 \mathrm{p}$.

Schuh, R. T. y J.A Slater, J. A. 1995. True bugs of the world (Hemiptera: Heteroptera). Classification and natural history. Cornell University Press, Ithaca, New York. p. 220-225. 